\title{
Maass operators and van der Pol-type identities for Ramanujan's tau function
}

\author{
by
}

\section{Dominic LANPHIER (Manhattan, KS)}

1. Introduction. For $z$ in the upper half plane $\mathcal{H}$ let $e(z)=e^{2 \pi i z}$. Ramanujan's tau function $\tau(n)$ is then defined by the expansion

$$
\Delta(z)=e(z) \prod_{n=1}^{\infty}(1-e(n z))^{24}=\sum_{n=1}^{\infty} \tau(n) e(n z),
$$

where $\Delta(z)$ is the cuspform of weight 12 and level 1 . Using differential equations satisfied by $\Delta(z)$, Eisenstein series, and certain other functions van der Pol [9] (and Resnikoff in [10]) established identities relating $\tau(n)$ to sum-of-divisors functions. For example, van der Pol showed

$$
\tau(n)=n^{2} \sigma_{7}(n)-540 \sum_{m=1}^{n-1} m(n-m) \sigma_{3}(m) \sigma_{3}(n-m)
$$

where $\sigma_{k}(n)=\sum_{d \mid n} d^{k}$.

In this paper we use Maass operators (see [7])

$$
\delta_{\kappa}=\frac{1}{2 \pi i}\left(\frac{\kappa}{2 i y}+\frac{\partial}{\partial z}\right)
$$

to prove a number of similar identities relating Ramanujan's tau function to sum-of-divisors functions, and in particular we establish the van der Pol identities in a natural way. Our method is analogous to the classical method of establishing identities among Fourier coefficients of modular forms of low weight. That is, for $E_{\kappa}(z)$ the normalized Eisenstein series of weight $\kappa$ and level 1 , we have relations like

$$
E_{4}(z) E_{8}(z)=E_{12}(z)+\frac{432000}{691} \Delta(z)
$$

and from (1) we can obtain identities for $\tau(n)$. Here we study the explicit structure of the non-holomorphic modular form $\delta_{\kappa}^{(q)} E_{\kappa}(z) \cdot \delta_{\mu}^{(r)} E_{\mu}(z)$ and

2000 Mathematics Subject Classification: Primary 11F30; Secondary 11F25. 
obtain twelve identities for $\tau(n)$ (essentially) including van der Pol's identities. These methods can of course be applied to the Fourier coefficients of other modular forms, but for simplicity we restrict our interest to $\tau(n)$ and holomorphic Eisenstein series. Some of these ideas were studied in [6] and applied to special values of $L$-functions.

We classify the identities into four theorems, based on the appearance of $\sigma_{k}$ 's in the summations. Some identities in each theorem are equivalent to each other, while some follow from the others in the theorem using elementary methods and classical identities of sum-of-divisors functions.

THEOREM 1.

$$
\begin{aligned}
& \text { (i) } \tau(n)=n^{2} \sigma_{7}(n)-540 \sum_{m=1}^{n-1} m(n-m) \sigma_{3}(m) \sigma_{3}(n-m), \\
& \text { (ii) } \tau(n)=-\frac{5}{4} n^{2} \sigma_{7}(n)+\frac{9}{4} n^{2} \sigma_{3}(n)+540 \sum_{m=1}^{n-1} m^{2} \sigma_{3}(m) \sigma_{3}(n-m), \\
& \text { (iii) } \tau(n)=n^{2} \sigma_{7}(n)-\frac{1080}{n} \sum_{m=1}^{n-1} m^{2}(n-m) \sigma_{3}(m) \sigma_{3}(n-m), \\
& \text { (iv) } \tau(n)=-\frac{1}{2} n^{2} \sigma_{7}(n)+\frac{3}{2} n^{2} \sigma_{3}(n)+\frac{360}{n} \sum_{m=1}^{n-1} m^{3} \sigma_{3}(m) \sigma_{3}(n-m) .
\end{aligned}
$$

Note that (i) and (ii) are equivalent and (iii) and (iv) are equivalent. Identity (i) is equation (2), essentially proven in [9] but with an error, corrected in [10].

Theorem 1 yields the congruences

$$
\tau(n) \equiv n^{2} \sigma_{7}(n)(\bmod 540),
$$

which is congruence (7.3) from [5], and

$$
\tau(n) \equiv n^{2} \sigma_{3}(n)(\bmod 240),
$$

which improves a congruence from [9].

THEOREM 2.

$$
\begin{aligned}
& \text { (i) } \tau(n)=-\frac{11}{24} n \sigma_{9}(n)+\frac{35}{24} n \sigma_{5}(n)+350 \sum_{m=1}^{n-1}(n-m) \sigma_{3}(m) \sigma_{5}(n-m), \\
& \text { (ii) } \tau(n)=\frac{11}{36} n \sigma_{9}(n)+\frac{25}{36} n \sigma_{3}(n)-350 \sum_{m=1}^{n-1} m \sigma_{3}(m) \sigma_{5}(n-m), \\
& \text { (iii) } \tau(n)=\frac{1}{6} n \sigma_{9}(n)+\frac{5}{6} n \sigma_{3}(n)-\frac{420}{n} \sum_{m=1}^{n-1} m^{2} \sigma_{3}(m) \sigma_{5}(n-m),
\end{aligned}
$$


(iv) $\quad \tau(n)=n \sigma_{9}(n)-\frac{2100}{n} \sum_{m=1}^{n-1} m(n-m) \sigma_{3}(m) \sigma_{5}(n-m)$,

(v) $\tau(n)=-\frac{1}{4} n \sigma_{9}(n)+\frac{5}{4} n \sigma_{5}(n)+\frac{300}{n} \sum_{m=1}^{n-1}(n-m)^{2} \sigma_{3}(m) \sigma_{5}(n-m)$.

It is easy to see that (i) and (ii) are equivalent and (iii), (iv), and (v) are equivalent.

From Theorem 2 we get the congruences

$$
\begin{array}{ll}
\tau(n) \equiv n \sigma_{9}(n)(\bmod 2100) & \text { for } 2,3,5,7 \nmid n, \\
\tau(n) \equiv n \sigma_{5}(n)(\bmod 240) & \text { for } 2,3,5,7 \nmid n, \\
\tau(n) \equiv n \sigma_{3}(n)(\bmod 504) & \text { for } 2,3,5,7 \nmid n .
\end{array}
$$

Combining these and a previous congruence gives

$$
(n-1) \sigma_{3}(n) \equiv 0(\bmod 24) \quad \text { for } 2,3,5,7 \nmid n \text {. }
$$

A catalog of similar congruences for $\sigma_{k}$ 's and $\tau(n)$ is given in [4] and [5].

THEOREM 3.

$$
\tau(n)=\frac{65}{756} \sigma_{11}(n)+\frac{691}{756} \sigma_{5}(n)-\frac{2 \cdot 691}{3 n} \sum_{m=1}^{n-1} m \sigma_{5}(m) \sigma_{5}(n-m) .
$$

For the last theorem we have $\sigma_{3}$ and $\sigma_{7}$ in the summations.

Theorem 4.

$$
\begin{aligned}
\tau(n)= & -\frac{91}{600} \sigma_{11}(n)+\frac{691}{600} \sigma_{3}(n)+\frac{4 \cdot 691}{5 n} \sum_{m=1}^{n-1} m \sigma_{3}(m) \sigma_{7}(n-m), \\
\tau(n)= & -\frac{91}{600} \sigma_{11}(n)+\frac{691}{600} \sigma_{7}(n) \\
& +\frac{2 \cdot 691}{5 n} \sum_{m=1}^{n-1}(n-m) \sigma_{3}(m) \sigma_{7}(n-m) .
\end{aligned}
$$

It is easy to see that (i) and (ii) here are equivalent.

As a consequence of these identities we get some other relations among the sum-of-divisors functions, such as

$$
\sum_{m=1}^{n-1} m(n-m)(n-2 m) \sigma_{3}(m) \sigma_{3}(n-m)=0 .
$$

2. Eisenstein series and Maass operators. In this section we introduce our Eisenstein series, Maass operators, and prove the key proposition. For

$$
\Gamma_{\infty}=\left\{\left(\begin{array}{cc}
1 & n \\
0 & 1
\end{array}\right) \mid n \in \mathbb{Z}\right\}
$$


denote the Eisenstein series of weight $\kappa$ and level 1 by

$$
E_{\kappa}(z)=\sum_{\left(\begin{array}{c}
a b \\
c d
\end{array}\right) \in \Gamma_{\infty} \backslash \mathrm{SL}_{2}(\mathbb{Z})}(c z+d)^{-\kappa}=1+\frac{2}{\zeta(1-\kappa)} \sum_{n=1}^{\infty} \sigma_{\kappa-1}(n) e(n z)
$$

where $\zeta(s)$ is the Riemann zeta function.

Let $\mathcal{M}_{\kappa}$ denote the space of modular forms of weight $\kappa$ and level 1 and let $\mathcal{C}_{\kappa}$ denote the subspace of cuspforms. Consider the differential operator

$$
\delta_{\kappa}^{(r)}=\left(\frac{1}{2 \pi i}\right)^{r}\left(\frac{\kappa+2 r-2}{2 i y}+\frac{\partial}{\partial z}\right)\left(\frac{\kappa+2 r-4}{2 i y}+\frac{\partial}{\partial z}\right) \ldots\left(\frac{\kappa}{2 i y}+\frac{\partial}{\partial z}\right)
$$

where $\delta_{\kappa}^{(0)}$ is just the identity operator and $z=x+i y$. Note that from Maass [7] the operator $\delta_{\kappa}^{(r)}$ preserves automorphy of $g_{\kappa}(z) \in \mathcal{M}_{\kappa}$ but not holomorphy. Further, note that if $g_{\kappa}(z) \in \mathcal{M}_{\kappa}$ then $\delta_{\kappa}^{(r)} g_{\kappa}(z)$ is a nonholomorphic modular form of weight $\kappa+2 r$ (see [2] and [3]).

We now study the structure of $\delta_{\kappa}^{(q)} E_{\kappa}(z) \cdot \delta_{\mu}^{(r)} E_{\mu}(z)$.

From [1] and [8] the action of $\delta_{\kappa}^{(r)}$ on $g_{\kappa}(z)=\sum_{n=0}^{\infty} a_{n} e(n z)$ is given explicitly by

where

$$
\delta_{\kappa}^{(r)} g_{\kappa}(z)=\sum_{n=0}^{\infty} a_{n}\left(\sum_{j=0}^{r} P_{j, \kappa}^{(r)}(-4 \pi y)^{-j} n^{r-j}\right) e(n z)
$$

$$
P_{j, \kappa}^{(r)}=\left(\begin{array}{c}
r \\
j
\end{array}\right) \frac{\Gamma(\kappa+r)}{\Gamma(\kappa+r-j)}
$$

and $\Gamma(s)$ is the usual gamma function.

The following is a lemma in [11], and see [12] for a more general discussion of nearly holomorphic modular forms and differential operators.

LEMMA 1 (Shimura). Let $G(z)$ be a function on $\mathcal{H}$ so that for $\gamma=$ $\left(\begin{array}{ll}a & b \\ c & d\end{array}\right) \in \mathrm{SL}_{2}(\mathbb{Z})$ we have

$$
G(\gamma(z))=(c z+d)^{\kappa} G(z) \quad \text { and } \quad G(z)=\sum_{j=0}^{r} a_{j} y^{-j} g_{\kappa-2 j}(z)
$$

where $g_{\kappa-2 j}(z)$ is holomorphic on $\mathcal{H}$ and has a Fourier expansion. Then

where $\widetilde{g}_{\kappa-2 j}(z) \in \mathcal{M}_{\kappa-2 j}$.

$$
G(z)=\sum_{j=0}^{r} \widetilde{a}_{j} \delta_{\kappa-2 j}^{(j)} \widetilde{g}_{\kappa-2 j}(z)
$$

As a consequence of Lemma 1 , for $g_{\kappa}(z) \in \mathcal{M}_{\kappa}$ and $g_{\mu}(z) \in \mathcal{M}_{\mu}$ we have the decomposition

$$
\delta_{\kappa}^{(q)} g_{\kappa}(z) \cdot \delta_{\mu}^{(r)} g_{\mu}(z)=\sum_{l=0}^{q+r} \delta_{\kappa+\mu+2 q+2 r-2 l}^{(l)}\left(\alpha_{l} E_{\kappa+\mu+2 q+2 r-2 l}(z)+\beta_{l} F(l, z)\right)
$$

where $F(l, z) \in \mathcal{C}_{\kappa+\mu+2 q+2 r-2 l}$ and $\alpha_{l}, \beta_{l} \in \mathbb{C}$. 
Proposition 1. For $q \leq r$,

$$
\begin{aligned}
\delta_{\kappa}^{(q)} E_{\kappa}(z) \cdot \delta_{\mu}^{(r)} E_{\mu}(z)= & \frac{\Gamma(\kappa+q) \Gamma(\mu+r) \Gamma(\kappa+\mu)}{\Gamma(\kappa) \Gamma(\mu) \Gamma(\kappa+\mu+q+r)} \delta_{\kappa+\mu}^{(q+r)} E_{\kappa+\mu}(z) \\
& +\sum_{l=0}^{q+r} \beta_{l} \delta_{\kappa+\mu+2 q+2 r-2 l}^{(l)} F(l, z)
\end{aligned}
$$

where the $F(l, z) \in \mathcal{C}_{\kappa+\mu+2 r+2 r-2 l}$ are normalized so their first non-zero Fourier coefficients are 1 and the $\beta_{l} \in \mathbb{C}$ consist of integer values of $\Gamma$ functions and $\zeta(s)$.

Proof. For simplicity write $E_{\kappa}(z)=\sum_{m=0}^{\infty} c_{\kappa}(m) e(m z)$; then from equation (3) we get

$$
\delta_{\kappa}^{(q)} E_{\kappa}(z)=\sum_{m=0}^{\infty} c_{\kappa}(m)\left(\sum_{k=0}^{q} P_{k, \kappa}^{(q)}(-4 \pi y)^{-k} m^{q-k}\right) e(m z) .
$$

For $E_{\mu}(x)=\sum_{n=0}^{\infty} c_{\mu}(n) e(n z)$ we then have

$$
\begin{aligned}
= & \left(\sum_{m=0}^{\infty} c_{\kappa}(m)\left(\sum_{k=0}^{q} P_{k, \kappa}^{(q)}(-4 \pi y)^{-k} m^{q-k}\right) e(m z)\right) \\
& \times\left(\sum_{n=0}^{\infty} c_{\mu}(n)\left(\sum_{j=0}^{r} P_{j, \mu}^{(r)}(-4 \pi y)^{-j} n^{r-j}\right) e(n z)\right) \\
= & \sum_{t=0}^{\infty} \sum_{s=0}^{q+r}\left(\sum_{m+n=t}\left(\sum_{j+k=s} c_{\kappa}(m) c_{\mu}(n) P_{k, \kappa}^{(q)} m^{q-k} P_{j, \mu}^{(r)} n^{r-j}\right)(-4 \pi y)^{-s}\right) e(t z) .
\end{aligned}
$$

For simplicity again, put

$$
E_{\kappa+\mu+2 q+2 r-2 l}(z)=\sum_{n=0}^{\infty} c(l, n) e(n z), \quad F(l, z)=\sum_{n=1}^{\infty} d(l, n) e(n z)
$$

where we normalize $F(l, z)$ so its first non-zero Fourier coefficient is 1 . Then

$$
\delta_{\kappa+\mu+2 q+2 r-2 l}^{(l)} E_{\kappa+\mu+2 q+2 r-2 l}(z)=\sum_{t=0}^{\infty} c(l, t)\left(\sum_{j=0}^{l} p_{j}^{l}(t) y^{-j}\right) e(t z)
$$

where $p_{j}^{l}(t)=P_{j, \kappa+\mu+2 q+2 r-2 l}^{(l)}(-4 \pi)^{-j} t^{l-j}$ and

$$
\delta_{\kappa+\mu+2 q+2 r-2 l}^{(l)} F(l, z)=\sum_{t=1}^{\infty} d(l, t)\left(\sum_{j=0}^{l} p_{j}^{l}(t) y^{-j}\right) e(t z) .
$$


Taking the Fourier expansions and switching the order of summations yields

$$
\begin{aligned}
\sum_{l=0}^{q+r} \delta_{\kappa+\mu}^{(l)} & \\
= & \sum_{l=0}^{q+r}\left[\alpha_{l} \sum_{t=0}^{\infty} c(l, t)\left(\sum_{s=0}^{l} p_{s}^{l}(t) y^{-s}\right) e(t z)\right. \\
& \left.+\beta_{l} \sum_{t=1}^{\infty} d(l, t)\left(\sum_{s=0}^{l} p_{s}^{l}(t) y^{-s}\right) e(t z)\right] \\
= & \sum_{s=0}^{q+r}\left(\sum_{l=s}^{q+r} \alpha_{l} c(l, 0) p_{s}^{l}(0)\right) y^{-s} \\
& +\sum_{t=1}^{\infty}\left[\sum_{s=0}^{q+r}\left(\sum_{l=s}^{q+r}\left(\alpha_{l} c(l, t) p_{s}^{l}(t)+\beta_{l} d(l, t) p_{s}^{l}(t)\right)\right) y^{-s}\right] e(t z)
\end{aligned}
$$

From Lemma 1 we can set the above result equal to (4). Then for $t=0$ we set the terms indexed by $(-4 \pi y)^{-s}$ equal and get the equation

$$
\left.\sum_{j+k=s}\left(P_{k, \kappa}^{(q)} m^{q-k} P_{j, \mu}^{(r)} n^{r-j}\right)\right|_{\substack{m=0 \\ n=0}}=\sum_{l=j+k}^{q+r} \alpha_{l} p_{j+k}^{l}(0) .
$$

Now,

$$
p_{j+k}^{j+k}(0)=(-4 \pi)^{-j-k} \frac{\Gamma(\kappa+\mu+2 q+2 r-j-k)}{\Gamma(\kappa+\mu+2 q+2 r-2 j-2 k)} \neq 0
$$

and $p_{j+k}^{l}(0)=0$ for $l \neq j+k$. Therefore equation (5) becomes

$$
\left.\left.\left(P_{k, \kappa}^{(q)} m^{q-k}\right)\right|_{m=0}\left(P_{j, \mu}^{(r)} n^{r-j}\right)\right|_{n=0}=\alpha_{j+k} p_{j+k}^{j+k}(0) .
$$

$\left.\operatorname{But}\left(P_{k, \kappa}^{(q)} m^{q-k}\right)\right|_{m=0}=0$ for $k \neq q$ and $P_{q, \kappa}^{(q)}=\Gamma(\kappa+q) / \Gamma(\kappa) \neq 0$, and similarly for $\left.\left(P_{j, \mu}^{(r)} n^{r-j}\right)\right|_{n=0}$. This implies $\alpha_{s}=0$ for $s<q+r$ and also

$$
\alpha_{q+r}=\frac{\left.\left.\left(P_{k, \kappa}^{(q)} m^{q-k}\right)\right|_{\substack{k=q \\ m=0}}\left(P_{j, \mu}^{(r)} n^{r-j}\right)\right|_{\substack{j=r \\ n=0}}}{p_{q+r}^{q+r}(0)}=\frac{\Gamma(\kappa+q) \Gamma(\mu+r) \Gamma(\kappa+\mu)}{\Gamma(\kappa) \Gamma(\mu) \Gamma(\kappa+\mu+q+r)} .
$$

For $t=1$ we set the $(-4 \pi y)^{-s} e(z)$ terms equal. As $c_{\kappa}(1)=2 / \zeta(1-\kappa)$ and $c_{\mu}(1)=2 / \zeta(1-\mu)$, we get the equation

$$
\begin{aligned}
\left.\sum_{j+k=s} \frac{2}{\zeta(1-\mu)}\left(P_{k, \kappa}^{(q)} m^{q-k}\right)\right|_{m=0} P_{j, \mu}^{(r)}+\left.\frac{2}{\zeta(1-\kappa)} P_{k, \kappa}^{(q)}\left(P_{j, \mu}^{(r)} n^{r-j}\right)\right|_{n=0} \\
=\sum_{l=j+k}^{q+r} \alpha_{l} c(l, 1) p_{j+k}^{l}(1)+\beta_{l} d(l, 1) p_{j+k}^{l}(1) .
\end{aligned}
$$


In a similar way to what happened for (5), equation (6) gives us

$$
\begin{aligned}
\sum_{l=s}^{q+r} \beta_{l} d(l, 1) p_{s}^{l}(1)= & -\alpha_{q+r} c(q+r, 1) p_{s}^{q+r}(1) \\
& +\left\{\begin{array}{cc}
0,2>s, & q>s \geq q, \\
\left.\frac{2}{\zeta(1-\mu)}\left(P_{k, \kappa}^{(q)} m^{q-k}\right)\right|_{\substack{k=q \\
m=0}} P_{s-q, \mu}^{(r)}, & r>s) \\
\left.\frac{2}{\zeta(1-\mu)}\left(P_{k, \kappa}^{(q)} m^{q-k}\right)\right|_{\substack{k=q \\
m=0}} P_{s-q, \mu}^{(r)} \\
+\left.\frac{2}{\zeta(1-\kappa)} P_{s-r, \kappa}^{(q)}\left(P_{j, \mu}^{(r)} n^{r-j}\right)\right|_{\substack{j=r \\
n=0}}, \quad s \geq r,
\end{array}\right.
\end{aligned}
$$

and evaluating these, we get

$$
\begin{aligned}
\sum_{l=s}^{q+r} \beta_{l} d(l, 1) p_{s}^{l}(1)= & -\alpha_{q+r} c(q+r, 1) p_{s}^{q+r}(1) \\
& + \begin{cases}0, & q>s, \\
\frac{2}{\zeta(1-\mu)}\left(\begin{array}{c}
r \\
s-q
\end{array}\right) \frac{\Gamma(\kappa+q) \Gamma(\mu+r)}{\Gamma(\kappa) \Gamma(\kappa+q+r-s)}, & r>s \geq q, \\
\left.\frac{2}{\zeta(1-\mu)}\left(\begin{array}{c}
r \\
s-q
\end{array}\right)+\frac{2}{\zeta(1-\kappa)}\left(\begin{array}{c}
q \\
s-r
\end{array}\right)\right] & s \geq r .\end{cases}
\end{aligned}
$$

Substituting $\alpha_{q+r}$ and the equation above into equation (6) we can solve for the $\beta_{l}$.

In order to illustrate an application of this result note that from Proposition 1 we have

$$
E_{4}(z) \cdot \delta_{4} E_{4}(z)=\frac{1}{2} \delta_{8} E_{8}(z)
$$

Setting the Fourier coefficients of the $(-4 \pi y)^{-1} e(n z)$ terms from both sides of this equation equal we get

$$
n \sigma_{7}(n)=n \sigma_{3}(n)+240 \sum_{m=1}^{n-1} m \sigma_{3}(m) \sigma_{3}(n-m) \text {. }
$$

This is formula (7.5) from [4].

3. Proofs of the identities for Ramanujan's tau function. We give a proof for Theorem 1. The proofs for the other theorems are similar and we give appropriate indications for those. From Proposition 1 we have 
(7) $\quad \delta_{4}^{(q)} E_{4}(z) \cdot \delta_{4}^{(r)} E_{4}(z)$

$$
=\frac{\Gamma(4+q) \Gamma(4+r) \Gamma(8)}{\Gamma(4)^{2} \Gamma(8+q+r)} \delta_{8}^{(q+r)} E_{8}(z)
$$

$+\beta_{0} \Phi_{8+2 q+2 r}(z)+\beta_{1} \delta_{6+2 q+2 r}^{(1)} \Phi_{6+2 q+2 r}(z)+\ldots+\beta_{q+r-2} \delta_{12}^{(q+r-2)} \Delta(z)$.

LEMMA 2.

$$
\begin{aligned}
\delta_{4} E_{4}(z) \cdot \delta_{4} E_{4}(z) & =\frac{2}{9} \delta_{8}^{(2)} E_{8}(z)-\frac{320}{3} \Delta(z), \\
E_{4}(z) \cdot \delta_{4}^{(2)} E_{4}(z) & =\frac{5}{18} \delta_{8}^{(2)} E_{8}(z)+\frac{320}{3} \Delta(z), \\
\delta_{4}^{(2)} E_{4}(z) \cdot \delta_{4} E_{4}(z) & =\frac{1}{9} \delta_{8}^{(3)} E_{8}(z)-\frac{160}{3} \delta_{12} \Delta(z), \\
E_{4}(z) \cdot \delta_{4}^{(3)} E_{4}(z) & =\frac{1}{6} \delta_{8}^{(3)} E_{8}(z)+160 \delta_{12} \Delta(z) .
\end{aligned}
$$

Proof. From (7) we have

$$
\begin{aligned}
\delta_{4} E_{4}(z) \cdot \delta_{4} E_{4}(z) & =\frac{2}{9} \delta_{8}^{(2)} E_{8}(z)+\beta_{0} \Delta(z), \\
E_{4}(z) \cdot \delta_{4}^{(2)} E_{4}(z) & =\frac{5}{18} \delta_{8}^{(2)} E_{8}(z)+\beta_{0} \Delta(z), \\
\delta_{4}^{(2)} E_{4}(z) \cdot \delta_{4} E_{4}(z) & =\frac{1}{9} \delta_{8}^{(3)} E_{8}(z)+\beta_{1} \delta_{12} \Delta(z), \\
E_{4}(z) \cdot \delta_{4}^{(3)} E_{4}(z) & =\frac{1}{6} \delta_{8}^{(3)} E_{8}(z)+\beta_{1} \delta_{12} \Delta(z) .
\end{aligned}
$$

We only need to look at the holomorphic part of each equation in order to solve for the $\beta_{j}$ 's. As

$$
\begin{aligned}
\delta_{4} E_{4}(z)= & \sum_{n=0}^{\infty} c_{4}(n)\left(n+4(-4 \pi y)^{-1}\right) e(n z), \\
\delta_{4}^{(2)} E_{4}(z)= & \sum_{n=0}^{\infty} c_{4}(n)\left(n^{2}+10(-4 \pi y)^{-1} n+20(-4 \pi y)^{-2}\right) e(n z), \\
\delta_{4}^{(3)} E_{4}(z)= & \sum_{n=0}^{\infty} c_{4}(n)\left(n^{3}+18(-4 \pi y)^{-1} n^{2}\right. \\
& \left.+90(-4 \pi y)^{-2} n+120(-4 \pi y)^{-3}\right) e(n z), \\
\delta_{8}^{(2)} E_{8}(z)= & \sum_{n=0}^{\infty} c_{8}(n)\left(n^{2}+18(-4 \pi y)^{-1} n+72(-4 \pi y)^{-2}\right) e(n z), \\
\delta_{8}^{(3)} E_{8}(z)= & \sum_{n=0}^{\infty} c_{8}(n)\left(n^{3}+30(-4 \pi y)^{-1} n^{2}\right. \\
& \left.+270(-4 \pi y)^{-2} n+720(-4 \pi y)^{-3}\right) e(n z)
\end{aligned}
$$


we can find explicitly the holomorphic part of $\delta_{4}^{(q)} E_{4}(z) \cdot \delta_{4}^{(r)} E_{4}(z)$. The $e(t z)$ term of the holomorphic part of $\delta_{4} E_{4}(z) \cdot \delta_{4} E_{4}(z)$ is

$$
\sum_{m+n=t} m c_{4}(m) n c_{4}(n)
$$

and for $t=1$ this is 0 . The $e(t z)$ term for the holomorphic part of $\frac{2}{9} \delta_{8}^{(2)} E_{8}(z)$ $+\beta_{0} \Delta(z)$ is $\frac{2}{9} t^{2} c_{8}(t)+\beta_{0} \tau(n)$. Setting $t=1$ we get

$$
\beta_{0}=-\frac{2}{9} c_{8}(1)=-\frac{2}{9} \frac{2}{\zeta(-7)}=-\frac{320}{3} .
$$

This gives (i).

The $e(t z)$ term for the holomorphic part of $E_{4}(z) \cdot \delta_{4}^{(2)} E_{4}(z)$ is

$$
\sum_{m+n=t} c_{4}(m) n^{2} c_{4}(n)
$$

The $e(t z)$ term for the holomorphic part of $\frac{5}{18} \delta_{8}^{(2)} E_{8}(z)+\beta_{0} \Delta(z)$ is $\frac{5}{18} t^{2} c_{8}(t)$ $+\beta_{0} \Delta(z)$. Setting $t=1$ we get

$$
\beta_{0}=c_{4}(1)-\frac{5}{18} c_{8}(1)=\frac{2}{\zeta(-3)}-\frac{5}{18} \frac{2}{\zeta(-7)}=\frac{320}{3},
$$

which gives (ii).

The $e(t z)$ term for the holomorphic part of $\delta_{4}^{(2)} E_{4}(z) \cdot \delta_{4} E_{4}(z)$ is

$$
\sum_{m+n=t} m^{2} c_{4}(m) n c_{4}(n) .
$$

This is 0 for $t=1$. The $e(t z)$ term for the holomorphic part of $\frac{1}{9} \delta_{8}^{(3)} E_{8}(z)+$ $\beta_{1} \delta_{12} \Delta(z)$ is $\frac{1}{9} t^{3} c_{8}(t)+\beta_{0} t \tau(t)$. Setting $t=1$ we get

$$
\beta_{1}=-\frac{1}{9} \frac{2}{\zeta(-7)}=-\frac{160}{3},
$$

which gives (iii).

The $e(t z)$ term for the holomorphic part of $E_{4}(z) \cdot \delta_{4}^{(3)} E_{4}(z)$ is

$$
\sum_{m+n=t} c_{4}(m) n^{3} c_{4}(n)
$$

The $e(t z)$ term for the holomorphic part of $\frac{1}{6} \delta_{8}^{(3)} E_{8}(z)+\beta_{1} \delta_{12} \Delta(z)$ is $\frac{1}{6} t^{3} c_{8}(t)+\beta_{1} t \tau(t)$. Setting $t=1$ we get

$$
\beta_{1}=c_{4}(1)-\frac{1}{6} c_{8}(1)=\frac{2}{\zeta(-3)}-\frac{1}{6} \frac{2}{\zeta(-7)}=160
$$

which gives (iv). 
Theorem 1 follows from Lemma 2 by uniqueness of Fourier coefficients. Looking at the $e(n z)$ term of the holomorphic part of (i) gives us

$$
\sum_{m=0}^{n} m c_{4}(m)(n-m) c_{4}(n-m)=\frac{2}{9} n^{2} c_{8}(n)-\frac{320}{3} \tau(n)
$$

so

$$
\begin{aligned}
\tau(n) & =\frac{3}{320} \frac{2}{9} \frac{2}{\zeta(-7)} n^{2} \sigma_{7}(n)-\frac{3}{320} \frac{4}{\zeta(-3)^{2}} \sum_{m=1}^{n-1} m(n-m) \sigma_{3}(m) \sigma_{3}(n-m) \\
& =n^{2} \sigma_{7}(n)-540 \sum_{m=1}^{n-1} m(n-m) \sigma_{3}(m) \sigma_{3}(n-m),
\end{aligned}
$$

which is (i) of Theorem 1. In a similar way we get (ii).

Looking at the $e(n z)$ term of the holomorphic part of (iii) gives us

$$
\sum_{m=0}^{n} m^{2} c_{4}(m)(n-m) c_{4}(n-m)=\frac{1}{9} n^{3} c_{8}(n)-\frac{160}{3} n \tau(n)
$$

so

$$
\begin{aligned}
\tau(n)= & \frac{3}{160} \frac{1}{9} \frac{2}{\zeta(-7)} n^{2} \sigma_{7}(n) \\
& -\frac{3}{160} \frac{4}{\zeta(-3)^{2}} \frac{1}{n} \sum_{m=1}^{n-1} m^{2}(n-m) \sigma_{3}(m) \sigma_{3}(n-m) \\
= & n^{2} \sigma_{7}(n)-\frac{1080}{n} \sum_{m=1}^{n-1} m^{2}(n-m) \sigma_{3}(n) \sigma_{3}(n-m),
\end{aligned}
$$

which is (iii) from Theorem 1. In a similar way we get (iv).

In the same way we can establish the following lemmas.

LEMMA 3.

$$
\begin{aligned}
\delta_{4} E_{4}(z) \cdot E_{6}(z) & =\frac{2}{5} \delta_{10} E_{10}(z)+\frac{1728}{5} \Delta(z), \\
E_{4}(z) \cdot \delta_{6} E_{6}(z) & =\frac{3}{5} \delta_{10} E_{10}(z)-\frac{1728}{5} \Delta(z), \\
\delta_{4}^{(2)} E_{4}(z) \cdot E_{6}(z) & =\frac{2}{11} \delta_{10}^{(2)} E_{10}(z)+288 \delta_{12} \Delta(z), \\
\delta_{4} E_{4}(z) \cdot \delta_{6} E_{6}(z) & =\frac{12}{55} \delta_{10}^{(2)} E_{10}(z)+\frac{288}{5} \delta_{12} \Delta(z), \\
E_{4}(z) \cdot \delta_{6}^{(2)} E_{6}(z) & =\frac{21}{55} \delta_{10}^{(2)} E_{10}(z)-\frac{2016}{5} \delta_{12} \Delta(z) .
\end{aligned}
$$

LEMMA 4.

$$
\delta_{6} E_{6}(z) \cdot E_{6}(z)=\frac{1}{2} \delta_{12} E_{12}(z)-\frac{381024}{691} \delta_{12} \Delta(z) .
$$


LEMMA 5.

$$
\begin{aligned}
& \delta_{4} E_{4}(z) \cdot E_{8}(z)=\frac{1}{3} \delta_{12} E_{12}(z)+\frac{144000}{691} \delta_{12} \Delta(z), \\
& E_{4}(z) \cdot \delta_{8} E_{8}(z)=\frac{2}{3} \delta_{12} E_{12}(z)+\frac{288000}{691} \delta_{12} \Delta(z) .
\end{aligned}
$$

From these lemmas we get Theorems 2, 3, and 4 just as was done for Theorem 1.

\section{References}

[1] J. E. Beineke, Renormalization of certain integrals defining triple product L-functions, Pacific J. Math. 203 (2002), 89-114.

[2] D. Bump, Automorphic Forms and Representations, Cambridge Stud. Adv. Math. 55, Cambridge Univ. Press, 1997.

[3] P. B. Garrett, Holomorphic Hilbert Modular Forms, Wadsworth-Brooks-Cole, 1990.

[4] D. B. Lahiri, On Ramanujan's function $\tau(n)$ and the divisor function $\sigma_{k}(n) . I$, Bull. Calcutta Math. Soc. 38 (1946), 193-206.

[5] - On Ramanujan's function $\tau(n)$ and the divisor function $\sigma_{k}(n)$. II, ibid. 39 (1947), 33-52.

[6] D. Lanphier, The trace of special values of L-functions attached to modular forms, submitted.

[7] H. Maass, Siegel Modular Forms and Dirichlet Series, Lecture Notes in Math. 216, Springer, 1971.

[8] T. Orloff, Special values and mixed weight triple products, Invent. Math. 90 (1987), 169-188.

[9] B. van der Pol, On a non-linear partial differential equation satisfied by the logarithm of the Jacobian theta-functions, with arithmetical applications. I, II, Indag. Math. 13 (1951), 261-271, 272-284.

[10] H. L. Resnikoff, On differential operators and automorphic forms, Trans. Amer. Math. Soc. 124 (1966), 334-346.

[11] G. Shimura, The special values of the zeta functions associated with cusp forms, Comm. Pure Appl. Math. 29 (1976), 783-804.

[12] - Differential operators, holomorphic projection, and singular forms, Duke Math. J. 76 (1994), 141-173.

Department of Mathematics

Kansas State University

Manhattan, KS 66506-2602, U.S.A.

E-mail: lanphier@math.ksu.edu 